\title{
Metodología para caracterización y análisis de impacto en el medio de egresados de Instituciones de Educación Superior (IES) ${ }^{*}$
}

\author{
José Eucario Parra-Castrillón ${ }^{\star *}$, Sandra Milena Arias-Giraldo ${ }^{\star * *}$
}

* Artículo de investigación

** Magíster en Educación, Magíster en Software Libre, profesor de tiempo completo, Facultad de Ingeniería,

Unisabaneta, Antioquia, Colombia. Correo electrónico: eucarioparra5@gmail.com

*** Especialista en Gerencia de la Comunicación con Sistemas de Información, coordinadora de egresados, Universidad de San Buenaventura, Medellín, Antioquia, Colombia. Correo electrónico: samya15@usbmed.edu.co

Recibido: 14 de septiembre del 2016 Aprobado: 22 de enero del 2017

Cómo citar este artículo: Parra-Castrillón, J. E., Arias-Giraldo, S. M. (2017). Metodología para caracterización y análisis de impacto en el medio de egresados de Instituciones de Educación Superior. Cooperativismo \& Desarrollo, 111(25), xx-xx. doi: https://doi. org/10.16925/co.v25i111.1875

\section{Resumen}

Propósito: este artículo es un producto de divulgación de la investigación "Caracterización de los egresados de la Universidad de San Buenaventura Medellín y análisis de su impacto en el medio", desarrollada, entre junio del 2014 y agosto del 2015, por un equipo interdisciplinar de profesionales de las áreas de ingeniería, arquitectura, psicología, educación, contaduría, derecho y comunicación. Descripción: los objetivos de la investigación se centraron en caracterizar a los egresados en aspectos personales, académicos y laborales, así como en analizar su impacto en los campos de actuación e influencia. Punto de vista: el presente artículo no pretende revelar los resultados de la investigación, dado que se constituye en información de interés interna para la institución. Proyecta evidenciar la propuesta metodológica que puede servir de referencia para la labor de seguimiento a graduados que realizan las IEs. Se busca así aportar a dos asuntos preponderantes: evitar reducir los estudios a la mera aplicación de una encuesta, e incentivar una metodología de investigación científica interdisciplinaria. Conclusiones: el método es relevante porque comprendió diversas fases e instrumentos. Se trató de una investigación mixta y multidimensional que recopiló información a través de técnicas cualitativas, cuantitativas y documentales, utilizando variadas fuentes de información; asimismo, la forma como se interpretó el tamaño real de la población representa una novedad para los estudios sobre egresados.

Palabras clave: caracterización de egresados, egresados, estudios de impacto, seguimiento a graduados, triangulación de datos. 


\title{
Characterization methodology and impact analysis in the medium of higher education institutions' graduates
}

\begin{abstract}
Purpose: This article is a product of the research "Graduates' of the Universidad de San Buenaventura in Medellín characterization and medium's impact analysis ", developed between June 2014 and August 2015, by an interdisciplinary team of professionals in the areas of engineering, architecture, psychology, education, accounting, law and communication. Description: The objectives of the research were to characterize the graduates in personal, academic and labor aspects, as well as to analyze their impact in their fields of action and influence. Point of view: This article does not pretend to reveal the results of the investigation, since they constitute information of internal interest for the institution. It aims at presenting a methodological proposal that can serve as reference point for the graduates' follow-up task performed by Higher Education Institutions. It therefore seeks to contribute in two preponderant issues: avoiding the reduction of such studies to the mere application of a survey, and encouraging an interdisciplinary scientific research methodology. Conclusions: The method is relevant because it comprises several phases and instruments. It comprised a mixed and multidimensional research that compiled information through qualitative, quantitative and documentary techniques, using various sources of information. Additionally, the way the real size of the population was interpreted represents a novelty for studies about graduates.
\end{abstract}

Keywords: characterization of graduates, graduates, impact studies, graduates' follow-up, data triangulation.

\section{Metodologia para caracterização e análise de impacto no meio de formandos de instituições de ensino superior}

\section{Resumo}

Propósito: este artigo é um produto de divulgação da pesquisa "Caracterização dos formandos da Universidad de Buenaventura Medellín e análise de seu impacto no meio", desenvolvida entre junho de 2014 e agosto de 2015, por uma equipe interdisciplinar de profissionais das áreas de engenharia, arquitetura, psicologia, educação, contabilidade, direito e comunicação. Descrição: os objetivos da pesquisa se focaram em caracterizar os formandos em aspectos pessoais, acadêmicos e profissionais, bem como em analisar seu impacto nos campos de atuação e influência. Ponto de vista: este artigo não pretende revelar os resultados da pesquisa, tendo em vista que se constitui em informação de interesse interna para a instituição. Projeta evidenciar a proposta metodológica que pode servir de referência para o trabalho de seguimento a formandos que as IEs realizam. Pretende-se, assim, contribuir para dois assuntos preponderantes: evitar reduzir os estudos à mera aplicação de um questionário e incentivar uma metodologia de pesquisa científica interdisciplinar. Conclusões: o método é relevante porque compreendeu diversas fases e instrumentos. Tratou-se de uma pesquisa mista e multidimensional que coletou informação por meio de técnicas qualitativas, quantitativas e documentais, utilizando várias fontes de informação; além disso, a forma como o tamanho real da população foi interpretada representa uma novidade para os estudos sobre formandos.

Palavras-chave: caracterização de formandos, formandos, estudos de impacto, seguimento a formandos, triangulação de dados. 


\section{Introducción}

Una propuesta metodológica para estudios sobre egresados de las instituciones de educación superior resulta pertinente, en razón a que la información que se obtiene posibilita el seguimiento a los graduados. Además, porque el conocimiento sobre su impacto en el medio ofrece insumos para evaluar la calidad y la pertinencia de los programas académicos, los cuales se justifican desde sus aportes al desarrollo de la sociedad.

Evidentemente, la creación y el desarrollo de programas académicos necesita una estrecha relación con los contextos externos y apuntar en dirección a los intereses y necesidades del entorno. Las funciones mismas de las IEs - docencia, investigación, proyección social e internacionalización-, deben mantener coherencia con el medio, de modo que, efectivamente, el conocimiento encuentre validaciones externas, nutra debates públicos, tenga aplicación en las organizaciones y sirva de marco para interpretaciones objetivas de la realidad.

En concordancia con lo anterior, una de las necesidades de la academia es disminuir las brechas que separan los perfiles de las carreras profesionales y las realidades del contexto. Esto es, hasta dónde y en qué grado se están cumpliendo las aspiraciones de impacto social de los programas académicos, en qué medida se evidencia su pertinencia, y cómo y cuándo los egresados, las investigaciones, los programas de extensión o las experiencias derivadas de las relaciones internacionales tienen aplicabilidad en la sociedad.

Sin duda, un asunto fundamental para los programas de pregrado y posgrado es el conocimiento sobre su impacto en el entorno, en términos de desempeño de los egresados, la percepción social sobre el programa, la calidad de los docentes y la pertinencia curricular. En otras palabras, comparar los fundamentos de los proyectos educativos con los movimientos externos en el medio de actuación de los graduados. Tales necesidades justifican la realización de estudios de egresados como los expuestos en Schomburg (2004); Negrini y Varela (2004); Jaramillo, Giraldo y Ortiz (2006); Maya y Herrera (2011); y la Pontificia Universidad Javeriana (2012).

Uno de los propósitos de este tipo de estudios es la observación de las diferencias entre los objetivos de formación de los programas académicos y las realidades de los entornos empresariales y sociales. En este sentido, los sistemas de calidad de la educación incluyen dentro de sus guías factores asociados al seguimiento de egresados (CNA, 2013; 2014).
Cabe señalar que una situación problemática en las investigaciones sobre egresados es lo referente al método, especialmente por particularidades como las siguientes: si un programa académico ha tenido distintas promociones, cómo definir su entorno y sobre cuáles bases identificar sus fronteras; si la movilidad de los egresados es permanente, cómo obtener actualización de sus datos; si hay certezas sobre el número de graduados, pero no sobre su ubicación y estado actual, cómo establecer el tamaño de la población para fines estadísticos; y, por último, si la vinculación de los egresados al contexto interno de la institución es apenas ocasional, hasta dónde puede determinarse que la población sea finita o infinita - en investigación cuantitativa, una población es infinita si no se tiene acceso a su información en su totalidad, o si se desconoce el número total-.

En los estudios sobre egresados, lo más adecuado para cada programa académico es el establecimiento de criterios en la delimitación de su medio, es decir, determinar cuáles son los conceptos que definen su entorno: cuáles evaluaciones o mediciones cualitativas o cuantitativas deben crearse a fin de conocer el impacto en el medio.

De los asuntos anteriores se plantean interrogantes como los siguientes:

- ¿Cómo evaluar el impacto en el medio de los programas académicos de las IEs?

- ¿Cómo evaluar la percepción social sobre la calidad de los programas?

- ¿Cuáles elementos deben caracterizarse para identificar el contexto de los programas académicos?

- ¿Cuál es el modelo de gestión de información más adecuado y cuáles deben ser los medios más efectivos para el seguimiento a los egresados de las instituciones de educación superior?

\section{Desarrollo de la propuesta metodológica}

Se deben diferenciar los propósitos de caracterizar a los egresados y analizar su impacto. En los procesos de investigación, la caracterización se asocia con eventos descriptivos para la identificación de componentes, acontecimientos, actores, actuaciones y el contexto de experiencia, hecho o actividades (Sánchez, 2011). Según la Real Academia de la Lengua, el término caracterizar se define como "determinar los atributos peculiares de alguien o de algo, de modo que claramente se distinga de los demás". 
Por su parte, el concepto de impacto está relacionado con la huella, impronta o señal que deja algo en concreto, o con el efecto producido en la opinión pública por algún evento (González y Calcetero, 2009); es decir, analizar un impacto social significa conocer la percepción general sobre un suceso o sobre su incidencia en la comunidad. No obstante, esta es una definición simple, dado que la realidad de un impacto social puede estar oculta dentro variables indirectas.

En la investigación realizada se determinaron variables y categorías sobre caracterización e impacto a partir de las relaciones conceptuales entre las capacidades humanas, las competencias y el desempeño profesional, y se exploraron algunos conceptos en Delors (1996), Nussbaum (2012), y Said (2012). Para el proyecto en particular, se definieron los siguientes objetivos específicos: (a) comparar el perfil de los egresados de la Universidad de San Buenaventura Medellín con las demandas laborales y sociales; (b) conocer el estado laboral de los egresados al momento de la graduación; (c) identificar y describir las características de los egresados en cuanto a variables personales, laborales y académicas; (d) identificar aportes de los egresados en los ámbitos socio-político, empresarial, deportivo, académico, científico y cultural; (e) analizar la percepción sobre los egresados por parte de empresas e instituciones públicas y privadas de Medellín y el Área Metropolitana; (f) identificar casos exitosos de egresados por su posición y desempeño en los campos social, político, empresarial, deportivo, académico, científico y cultural.

Con el fin de alcanzar los objetivos propuestos se aplicaron técnicas cualitativas, cuantitativas y documentales, en busca de información sobre caracterización e impacto de los egresados. Así, entonces, en cada estrategia de búsqueda utilizada e instrumento aplicado se tuvieron en cuenta las dos categorías generales mencionadas.

\section{Delimitación del estudio}

Se consideró la población de egresados de todos los programas académicos de la Universidad de San Buenaventura Medellín, en el nivel de pregrado, desde la primera cohorte de cada uno hasta el 2014. No obstante, los mecanismos y conceptos para elección de muestras y estratos dejaron abierta la posibilidad de adaptación a la naturaleza y circunstancias propias de cada programa académico. En el estudio se incluyeron, tanto los programas con oferta vigente, como aquellos que ya no tenían cohortes abiertas. Esto al definir que, en el conglomerado social, las realizaciones y características de los egresados no dependen de si los programas están o no vigentes en la oferta académica de la institución.

Igualmente, la delimitación espacial del proyecto no se circunscribió exclusivamente a Medellín y el Valle de Aburrá, sino que abarcó ámbitos más generales gracias a que el ámbito de actuación de los egresados está determinado por sus posibilidades profesionales, tanto en ámbitos locales, como regionales, nacionales e internacionales.

\section{Tipo de investigación}

Para el cumplimiento de los objetivos se consideró una investigación descriptiva. Según Hernández, Fernández y Baptista (2006, p. 102), "los estudios descriptivos buscan especificar las propiedades, las características y los perfiles de personas, grupos, comunidades, procesos, objetos o cualquier otro fenómeno que se someta a un análisis". Su propósito es la medición, evaluación o recolección de información sobre conceptos, dimensiones, características o componentes de los eventos a investigar. La presente investigación integró una metodología mixta, involucrando las técnicas: (a) documental: incluyó la revisión de documentos históricos y bases de datos de la universidad, el sistema de información administrado en la Oficina de Egresados de la institución y algunas bases de datos de entidades externas públicas y privadas; (b) cuantitativas: mediante la aplicación de más de 1000 encuestas, con el objetivo de medir las variables objeto de las indagaciones; (c) cualitativas: a través del desarrollo de entrevistas y grupos focales, con base en las categorías de indagación.

La elección del estudio mixto se produjo debido a que la aproximación al impacto de los egresados implica el conocimiento de datos sobre variables específicas, lecturas de los discursos sobre el sentir de los egresados y, además, tendencias e históricos que reposan en bases de datos. La combinación de las técnicas cualitativas y cuantitativas hace posible un recorrido metodológico con dos concepciones: la aplicación de la estadística que busca describir leyes, principios o tendencias generales y, por otra parte, la utilización de grupos focales y entrevistas que permitan entender el fenómeno social de los egresados desde su propia perspectiva y desde la forma como interactúan con el contexto (Galeano, 2011, p. 12-26). 


\section{Investigación cuantitativa.}

El método cuantitativo tiene como base la medición de características o variables del fenómeno social que se estudia, y supone la existencia de unos postulados sobre las relaciones entre esas variables, las cuales se derivan desde el marco conceptual y la especificidad del problema analizado y sigue una lógica deductiva (Bernal, 2006; Vivanco, 2005). En la caracterización de los egresados se propusieron variables relacionadas con atributos personales y profesionales: (a) características personales: localidad, sexo, edad, competencias, segundo idioma; y (b) características laborales: condiciones iniciales, condiciones actuales, salario, tipo de contrato, área de desempeño, tipo de empresa, experiencia.

Para la medición del impacto, se establecieron variables asociadas a realizaciones de los egresados después de graduados que tienen que ver con su incidencia en la comunidad y en el sector empresarial: (a) atributos o incidencias académicas e investigativas: publicaciones, reconocimientos académicos, proyectos desarrollados, participación en redes o en colectivos, participación en investigaciones u obtención de patentes; (b) atributos relacionados con aspectos socio-políticos, culturales y artísticos: premios, proyectos, participación en el diseño de políticas, cargos públicos $o$ en organizaciones no gubernamentales, relaciones internacionales, vinculación a redes socio-políticas, culturales y artísticas e iniciativas empresariales; (c) atributos relacionados con aspectos empresariales: emprendimiento, desempeño profesional y reconocimiento empresarial; (d) características relacionadas con la formación humana: grado de correspondencia con los ejes pedagógicos, antropológicos y psicológicos de la Paideia Franciscana (fundamento pedagógico de la Universidad de San Buenaventura).

\section{Investigación cualitativa.}

Este método permitió analizar el contenido del discurso a fin de evaluar asuntos relacionados con el impacto de los egresados en el medio, considerando dos técnicas, fundamentalmente: entrevistas y grupos focales. Esto permitió conocer las actuaciones, los desempeños y la percepción externa sobre los egresados (Tojar, 2006; Wolcott, 2006).

En el caso concreto del estudio, las técnicas de grupos focales y entrevistas hicieron posible la interpretación del contenido del discurso de los egresados, de los directores de programa y de la coordinación de egresados de la universidad, así como los sentimientos y las percepciones sobre las relaciones de los egresados con el contexto, sus expectativas y la forma como evalúan la formación profesional recibida. Especial importancia se dio a la aplicación de las técnicas cualitativas a las dimensiones humanas de la formación y a la incidencia de la misión católica de la Universidad de San Buenaventura.

Al contrario de la investigación cuantitativa, el diseño cualitativo es abierto y flexible. No se plantea una definición previa de variables, sino que se postulan categorías que se van construyendo en la medida en que avanza el análisis y la interpretación del contenido del discurso. En el caso específico del estudio, el mecanismo cualitativo permitió conocer la realidad de los egresados vista a partir de las múltiples lógicas y situaciones particulares de las distintas facultades de la universidad. La mencionada construcción de las categorías se realizó a partir de preguntas orientadoras para los diálogos en las entrevistas y los grupos focales:

- Concepto global sobre la calidad de la formación recibida en la universidad.

- Diferencias entre las expectativas de los estudiantes y las realidades laborales y profesionales.

- Logros obtenidos en lo político, empresarial, deportivo, académico, científico y cultural, luego de la graduación.

- Expresión de los valores y la formación humana que postula la universidad en su filosofía institucional (cómo se evidencian en el desarrollo profesional).

- Impronta de los egresados de la Universidad de San Buenaventura.

- Concepción sobre el aspecto religioso (al ser una institución católica).

- Criterio político y social del egresado frente al país.

- Percepción que sobre los egresados tienen los empleadores y, en general, el contexto social, así como el nivel de favorabilidad con respecto a egresados de otras universidades.

- Proyección y expectativas del egresado hacia su futuro.

- Sugerencias para la universidad.

\section{Investigación documental.}

Básicamente, se involucraron fuentes de información tales como: bases de datos de la Universidad de San Buenaventura Medellín; bases de datos de entidades externas privadas, gubernamentales o no gubernamentales; históricos de las facultades y programas 
académicos; e información del DANE y de la plataforma del Observatorio Laboral para la Educación Superior del Ministerio de Educación Nacional. Asimismo, se consultaron redes especializadas como Red de Enlace Profesional (2015), y Red GRadua2/ Asociación Columbus (2006).

\section{Fuentes}

Para la obtención de la información sobre características e impacto de los egresados, se tuvieron en cuenta las siguientes fuentes:

- Egresados de todos los programas académicos de la universidad, vigentes o no en la oferta.

- Directores de los programas académicos.

- Bases de datos de la universidad.

- Ejecutivos del sector empresarial.

- Observatorio Laboral para la Educación Superior del Ministerio de Educación Nacional.

\section{Consideraciones especiales para determinar el tamaño de la población}

Se determinó el número de graduados hasta febrero del 2014 - fecha de corte-, momento en el cual el número de egresados sumaba 19 458. Sin embargo, el tamaño real de la población para la aplicación del formulismo estadístico fue de 9501 egresados, en virtud de la estratificación y el muestreo, además, considerando el número de egresados a los que potencialmente se pudo acceder mediante información de contacto vigente.

Las siguientes condiciones llevaron a la determinación sobre el valor real de la población de estudio: (a) del número total de egresados no se conoce cuantos han fallecido, ni cuántos se encuentran desconectados de los sistemas de información o comunicación que facilitan el contacto con la institución; (b) al momento de la graduación de muchos egresados no existían mecanismos de contacto como el correo electrónico o teléfonos celulares, lo que hace que se tenga una proporción de egresados sin información de contacto; (c) la población de egresados se distribuye en un entorno geográfico muy amplio, y es natural su movilidad residencial o laboral; (d) el seguimiento en tiempo real es complejo, dado que, aunque tecnológicamente es posible el establecimiento de contacto, depende en gran medida de la autodeterminación y la voluntad por parte de los egresados; (e) los egresados cada vez están más concentrados en su entorno (trabajo, negocios, familia) que en el contexto de la universidad, es decir, los intereses varían en relación con la vida inmediata del egresado; (f) las bases de datos son cada vez más restringidas debido a la Ley de Habeas Data, de ahí que resulta compleja la consulta de información de contacto de los egresados, en archivos públicos o privados.

En suma, se estableció cómo tamaño real de la población (9501 egresados), en razón a la información registrada en la Oficina de Egresados de la Universidad; el criterio fue el acceso a los correos electrónicos y teléfonos de contacto.

\section{Encuesta aplicada al sector empresarial para análisis de impacto}

Como parte de los instrumentos, se aplicó una encuesta al sector empresarial que respondieron 89 ejecutivos. Los objetivos trazados fueron los siguientes: (a) conocer la percepción sobre las competencias profesionales y personales de los egresados de la Universidad de San Buenaventura Medellín; (b) identificar el nivel de favorabilidad de los egresados por parte de las empresas; (c) descubrir apreciaciones con respecto a las actitudes y formación humana de los egresados.

La muestra de 89 empresas fue de tipo causal, y obedeció al único criterio de tener en la organización a uno o varios egresados de la universidad. El propósito en la aplicación de este instrumento estuvo relacionado con la medición del impacto de los egresados, en razón a su nivel de aceptación en las organizaciones y a las percepciones que pueden tenerse sobre sus competencias. La muestra se seleccionó de manera arbitraria, considerando que el funcionario que respondiera los interrogantes tuviera como referencia a algún egresado que laborara en su misma área de trabajo, por cuanto es más factible dar cuenta del respectivo desempeño. Vale anotar que no se realizó estratificación por tipo de empresa o localidad geográfica, ni se aplicaron técnicas estadísticas para la elección de la muestra; sin embargo, se definió que la cifra de 89 empresas es suficiente para generalizar los resultados obtenidos debido a la naturaleza de las preguntas. Lógicamente, no se puede descartar algún posible sesgo, independientemente de si se hubiera aplicado o no estratificación y muestreo, ya que las preguntas se plantearon con base en generalidades del conjunto de egresados.

Con relación a las preguntas dirigidas a evaluar el nivel de favorabilidad sobre los egresados de diferentes instituciones de educación superior de Medellín y el Área Metropolitana, el propósito estuvo encaminado 
a comparar cómo se ubica la Universidad de San Buenaventura Medellín en la mencionada variable. Lo anterior puede suministrar información sobre al impacto de los egresados, ya que los estudios sobre favorabilidad revelan la generalidad de una población cuya percepción puede resultar positiva o negativa.

Las instituciones seleccionadas para la indagación sobre favorabilidad fueron clasificadas en dos grupos, según los siguientes criterios: (a) Grupo 1: instituciones de educación superior tradicionales y arraigo histórico en el común de la gente; (b) Grupo 2: instituciones de educación superior emergentes y con cantidad creciente de estudiantes.

Vale anotar que en el capítulo de conclusiones y hallazgos del estudio se evitó relacionar el listado clasificatorio de las instituciones, pese a que se cuenta con los datos obtenidos con la encuesta aplicada. Los resultados de la mencionada variable de clasificación se presentaron según una evaluación de la percepción en la escala de 1 a 5 , promediada para cada uno de los grupos, comparándolos con el promedio que obtuvo la Universidad de San Buenaventura Medellín. Esto permitió establecer cuantitativamente la percepción sobre favorabilidad de los egresados de la institución con respecto a los dos grupos.

\section{Triangulación de los resultados}

La investigación involucró dos estrategias de triangulación para evaluar la confiabilidad de los resultados: una de fuentes de datos y otra metodológica. En la primera se efectuó una comparación de los resultados encontrados por medio de las distintas fuentes, y en la segunda se combinaron varios métodos a fin de obtener y analizar los datos sobre la realidad de los egresados (Arias, 2000).

Ambas comparaciones proyectaron conclusiones como las siguientes: (a) los resultados obtenidos con la encuesta aplicada a los egresados con respecto a la variable de empleo y salario coincidieron en buena proporción con las cifras del DANE, y del Observatorio Laboral paralaEducación del Ministerio de Educación Nacional; (b) los resultados de la encuesta aplicada al sector empresarial en cuanto al impacto de los egresados y las competencias profesionales, coincidieron en gran parte con la información obtenida en los grupos focales realizados con los egresados; (c) los aspectos relevantes de las entrevistas a los directores de los programas académicos coincidieron altamente con la información obtenida en los grupos focales con egresados, así como en la encuesta aplicada al sector empresarial; (d) los resultados obtenidos en la encuesta a los egresados sobre vinculación laboral, contratación y tipo de trabajo, coincidieron altamente con la información obtenida en los grupos focales y con la información disponible en las bases de datos de la Oficina de Egresados de la Universidad de San Buenaventura Medellín; respecto a la formación humana de los egresados y su relación con el Proyecto Educativo Bonaventuriano, se encontraron algunas diferencias entre las percepciones obtenidas a través de las encuestas a empresas y a egresados, con las expresadas en los grupos focales.

\section{Conclusiones}

La metodología aplicada en el proyecto fue suficiente para alcanzar los objetivos planteados sobre caracterización de los egresados y análisis de su impacto en el medio. Desde la metodología implementada y la triangulación de datos se logró validar la información obtenida por medio de los diferentes instrumentos aplicados.

Un estudio de egresados debe establecerse con el rigor de la investigación científica más que como proyecto de intervención, especialmente si se quiere obtener información multidimensional que abarque fuentes variadas e integralidad en la información. Así quedó evidenciado con la presente experiencia de la Universidad de San Buenaventura Medellín, gracias al proyecto interdisciplinario que posibilitó obtener un análisis completo acerca de la caracterización de los egresados y su impacto en el medio. Sin duda, al ser la población de egresados sumamente móvil, difusa y ubicada en contextos abiertos, resulta necesario implementar metodologías como la citada que, sin pretender abarcar a la totalidad de la población, pueda responder desde un enfoque mixto a las inquietudes de las instituciones de educación superior sobre la realidad de sus egresados.

De igual modo, el proyecto de egresados debe encuadrarse dentro de marcos contextuales y conceptuales que identifiquen aspectos de interés para la educación superior (en general, la institución, y en particular, el proceso curricular del programa académico), las demandas y normatividad del Ministerio de Educación Nacional, los elementos de las capacidades humanas y el capital humano, así como las nociones sobre los vínculos de la institución de educación superior con los intereses sociales y públicos.

Los proyectos sobre egresados deben incentivar espacios universitarios encaminados a la reflexión sobre pertinencia curricular, calidad de la formación 
profesional y fundamentación pedagógica y filosófica del proyecto educativo institucional.

Finalmente, las instituciones de educación superior deben concebir los proyectos de investigación sobre egresados y los procesos de seguimiento que se orientan desde las oficinas de egresados como pilares para la construcción y el rediseño curricular, así como un eje fundamental para el análisis de proyección social, extensión académica, internacionalización y mercadeo.

\section{Trabajos futuros}

El reto para investigaciones futuras es proponer un modelo universitario que permita activar vínculos entre los postulados misionales de las instituciones de educación superior y los objetivos de los procesos curriculares de los programas académicos, con la información sobre caracterización de egresados y las mediciones de impacto.

El propósito central sería el establecimiento de rutas a seguir para concretar un sistema de tipologías de información, a partir de las cuales pueda evaluarse, por una parte, la declaración misional de la institución y la calidad de los procesos curriculares, en función de la información sobre sus egresados y, por otra, la capacidad de impactar a la sociedad.

Otro objetivo para emprender una nueva investigación podría ser la construcción de memorias en las que queden consignados la biografía, los logros, las realizaciones de egresados sobresalientes en escenarios de lo empresarial, lo político, lo académico, lo artístico y lo deportivo. La citada memoria se constituiría en una buena representación del impacto de los egresados en el medio.

\section{Referencias}

Arias, M. (2000, marzo). La triangulación metodológica. Sus principios, alcances y limitaciones. Investigación y Educación en Enfermería. Medellín, XVIII(1).

Bernal, A. (2006). Metodología de la investigación para administración, economía, humanidades y ciencias sociales. México: Pearson Educación.

Consejo Nacional de Acreditación-CNA. (2013). Sistema Nacional de Acreditación. Lineamientos para la Acreditación Institucional 2015. Bogotá.

Consejo Nacionalde Acreditación-CNA. (2014).Lineamientos para la Acreditación de Programas de Pregrado. Bogotá.

Cullen, C. (2005). Perfiles ético-políticos de la educación. Barcelona: Paidós Iberia.

Delors, J. (1996). La educación encierra un tesoro. Madrid: Santillana Ediciones, Unesco.
Galeano, E. (2011). Diseño de proyectos de investigación cualitativa. Medellín: Fondo Editorial EAfIT.

González, C., y Calcetero, J. (2009, octubre). Evaluación de impacto social: una estrategia de investigación para trabajo social. Revista Tendencias \& Retos, 14, 43-57.

Hernández, R., Fernández, C., y Baptista, P. (2006). Metodología de la investigación. México: McGraw-Hill.

Jaramillo, A., Giraldo, A., y Ortiz, J. (2006). Estudios sobre egresados. La experiencia de la Universidad EAFIT. Revista Universidad EAFIT, 43, 111-124.

Maya, J., y Herrera, M. (2011, septiembre-diciembre). Caracterización e impacto de los graduados de la Católica del Norte Fundación Universitaria. Revista Virtual Universidad Católica del Norte, 34, 78-112.

Ministerio de Educación Nacional. Graduados Colombia Observatorio Laboral para la Educación. Recuperado de http://www.graduadoscolombia.edu.co/

Negrini, V., y Varela, G. (2004). Diagnóstico sobre el estado actual de los estudios de egresados. Delegación Benito Juárez, México: ANUies.

Nussbaum, M. (2012). Crear capacidades: propuesta para el desarrollo humano. Barcelona: Paidós.

Pontificia Universidad Javeriana. (2012). Estudio de seguimiento a recién egresados de programas académicos de pregrado de la sede central. Bogotá: Pontificia Universidad Javeriana

Red de Enlace Profesional. (2015). Escala salarial 2016 Red Enlace Profesional. Recuperado de http://www.enlaceprofesional. com.co/

Red GRAduA2/Asociación Columbus. (2006). Manual de instrumentos y recomendaciones sobre el seguimiento de egresados. Monterrey, México: Alfa.

Said, E. (Ed.). (2012). Habilidades cognitivas y socioemocionales: un estudio en estudiantes de media vocacional en estudiantes de media vocacional y formación técnica en el atlántico. Barranquilla: Editorial Universidad del Norte.

Sánchez, U. (2011). Manual de redacción académica e investigativa: cómo escribir, evaluar y publicar artículos. Medellín: Católica del Norte Fundación Universitaria.

Schomburg, H. (2004). Manual para estudios de seguimiento de graduados universitarios. Boon, Alemania: InWent.

Tojar, J. (2006). Investigación cualitativa. Comprender y actuar. Madrid: La Muralla S. A.

Vivanco, M. (2005). Muestreo estadístico. Diseño y aplicaciones. Santiago de Chile: Editorial Universitaria S.A.

Wolcott, H. (2006). Mejorar la escritura de la investigación cualitativa. Medellín: Editorial Universidad de Antioquia. 